\title{
The Sense of Coherence in the Life Course
}

\author{
Claudia Meier Magistretti
}

\section{Introduction}

Antonovsky understood the sense of coherence (SOC) as the outcome of learning processes that begin at the time of birth, or even earlier. To create a strong SOC, these processes should provide challenges that are perceived as consistent, that allow participation, and are characterised by an underload-overload balance. Initially, Antonovsky considered the SOC to be relatively stable after early adulthood, with only minor fluctuations occurring after that. Later, he clarified that stability of the SOC pertains mainly for adults with a strong SOC, since "the person who has, in early adulthood, crystallised a strong SOC, [has] the ability to bring into play the generalised resistance resources available to him or her" (Antonovsky, 1987, p. 121).

Antonovsky assumed that early childhood is a critical period to create a strong SOC. He conceptualised the infant as an active subject that can interact in ways that produce stable, consistent response interactions (Antonovsky, 1987, p. 94). These actions occur very early, according to Antonovsky, immediately after birth.

Relating to Bowlby's attachment theory, he developed the idea that a strong sense of comprehensibility is fostered early in life by the consistent experience of parents' and other caregivers' responsiveness and sensitivity. Still, Antonovsky criticised the imperative for parents to be always consistent and responsive, stating that real life is far more complex. Perfectly responsive parents do not exist, because multiple tasks and demanding environments are the reality for all. On the contrary, "natural" frustrations are necessary to create a sense of comprehensibility. When the infant learns that objects and satisfactions can disappear and reappear, he or she learns about the continuity (and to a certain extent the predictability) of social interactions.

C. Meier Magistretti $(\bowtie)$

Centre for Health Promotion and Participation, Lucerne University

of Applied Sciences and Arts, Lucerne, Switzerland

e-mail: claudia.meiermagistretti@hslu.ch
Manageability is, according to Antonovsky, built by experiences of load balance fostered by parents that respect their child's abilities at different stages of development. Using the example of forced toilet training in toddlers, Antonovsky describes how overloading demands are created when the biological and psychological realities of the child are not sufficiently recognised. Consequently, the development of the sense of manageability can be disturbed or hindered by inappropriate expectations of a child's caregivers. In Antonovsky's thinking, load balance is achieved when a demand corresponds to the child's development state, in the sense that it is somehow "reasonable" and when it is subjected to the child's choice.

The sense of meaningfulness develops by participation in decision-making, which in early childhood occurs when the infant experiences that his or her actions produce the desired reaction in the adult. "To the extent that desired outcome is contingent on the infant and child's action", Antonovsky stated, "it can reasonably be said that early on there is participation in decision making" (Antonovsky, 1985, p. 97). The sense of meaningfulness in the early stage of life is fostered by the infant's experience of being able to pressure the environment to act and thereby comprehend that she or he matters to the parents or other caregivers.

In adolescence, the SOC is potentially volatile, and the basis that has been laid in childhood for a strong the SOC may become upset. This stage of life is characterised by transitions and a constant balancing of a fragile identity between "not anymore" and "not yet". Antonovsky related the development of the SOC to Erik Erikson's main developmental tasks that adolescents have to face in all cultures:

- First, they are challenged to develop a personality within a social reality that they have to understand.

- Second, they have to "get their act together" to acquire a "vitalising sense of reality" (Erikson, 1950, p. 89).

- Third, they need growing experience mastering challenges. 
The sense of comprehensibility is reinforced by the growing ability of the adolescent to develop a sense of reality. The experience of mastering challenges nurtures the sense of manageability. The sense of meaningfulness is supported by a "vitalising" way to deal with reality. Still, Antonovsky appreciated that adolescence is a fragile period of life, needing adult guidance.

Adulthood is perceived as a period of life where roles and GRRs are decisive for the development of the SOC and for shaping identity. Antonovsky assumed that in the first decade of adulthood, an individual tends to put together the new roles he or she assumes at work, in partnership, in creating a family, and in society. While some of these new roles may weaken the individual's SOC, others may strengthen it. The SOC is assumed to remain somewhat volatile in this period of life. Antonovsky supposed (but gave indications of not being entirely convinced) that at the end of the second decade, individuals have sorted out or accepted the different areas of life, and "one has attained a given location on the SOC continuum" (Antonovsky, 1987, p. 119).

Following Antonovsky's passing in 1994, Lindström and Eriksson (2010) defined the development of the SOC as a lifelong learning process. Analysing SOC levels in different age groups in longitudinal studies, they observed the SOC's tendency to strengthen with age (Lindström \& Eriksson, 2010). The strengthening of the SOC over the lifespan isneglecting some fluctuations-continuous, consistent over different measurement scales (SOC-13, the SOC-29), steady, and moderate.

Nevertheless, we know little about how the development of a stronger SOC occurs. Only recently, Maas and colleagues (2017) investigated mechanisms strengthening the SOC in adults. They found that systematic, coherent experiences in everyday life might play a crucial role in building a strong SOC.

Significant life events may cause fluctuations in the SOC. Becoming a parent, for example, is posited to be s a critical life experience that may alter one's SOC (Lindström et al., 2017). From the perspective of anthropological pedagogics, we can hypothesise that the SOC, as any other learning outcome, could benefit from sensitive phases of development. These have been studied in children as a developmental phase of specific readiness to learn, which enables a person to acquire specific skills and characteristics relatively effortlessly (e.g. Montessori, 1995). Further investigation of the SOC as a lifelong learning process may reveal critical points throughout life when the SOC is particularly prone to change.

As the preceding discussion shows, the development of the SOC is understood as a lifelong learning process. Among the important questions about this process are as follows:
- What are the crucial factors that contribute to build, enhance, and strengthen the SOC during the life course?

- Is the role of the SOC in coping stable during the life course, or does it have different functions at different life stages? For example, does the SOC open one to new GRRs at the beginning and facilitate access to GRRs later in life?

- Can salutogenic interventions strengthen the SOC during various phases in the lifespan?

The chapters in this part of the Handbook tackle all three of these questions with varying emphasis at different periods of life.

\section{Childhood and Families}

Chapter 15 on salutogenesis and the sense of coherence in families and children deals with the contributions of children's environments to the adjustment of children with typical development and the development of children with special needs. The chapter starts with a short description of childhood development. Orly Idan and colleagues report on their comprehensive literature review covering 20 years of research and 44 studies from 15 countries, including children from infants and toddlers through preschool to school-aged children up to 12 years of age. The construction and use of the children's SOC scale and methodological considerations are discussed. Also in focus is the influence of the SOC on various health outcomes, and children's emotional, social, and cognitive development (both in children with regular development and in children facing challenging life circumstances, health-related adversity, and special needs).

The chapter concludes that the SOC plays a significant protective role at school and emphasises the crucial role of teachers' ability to engage with children in responsive and caring relationships. For families with children with special needs, the SOC is demonstrated to be a significant protective factor related to coping and hopeful thinking, as well as to parental adjustment and effective coping outcomes. The chapter also provides examples of programs to foster the SOC in young children, by promoting play and reinforcing self-worth in the first years of school.

\section{Adolescence}

In Chap. 16 by Braun-Lewensohn and colleagues, the focus is on salutogenesis and the sense of coherence during the adolescent years. The authors' approach is itself salutogenic, in the sense that they develop their arguments in line with a positive youth development perspective. Adolescents are appreciated as individuals eager to explore the world, to 
acquire competence, and to struggle with challenges and difficulties, rather than as a vulnerable group in need of risk prevention, cure, and treatment for maladaptive tendencies.

Still, the literature is replete with studies of the authors' review of more than 30 studies in 16 countries shows a significant role of the SOC for physical and mental health, health behaviour, social adaption, and academic or professional success of adolescents. This is demonstrated for the individual the SOC (in several studies measured by specific adolescent the SOC scales) and hypothesised for family and community the SOC. In adolescence, the SOC is a predictor for physical health and the use of medication in consequence.

The studies reviewed in this chapter confirm that a strong $\mathrm{SOC}$ is associated with reduced stress, and decreased internalising/externalising of problems. Related to health behaviours, adolescents with stronger SOC report a more healthy lifestyle as well as a better quality of life and well-being. The authors report on studies observing that a strong SOC in adolescence is associated with higher levels of physical activity and exercise, less tobacco and alcohol use, and healthier eating habits.

Factors strengthening the SOC in adolescence are discussed, with an emphasis on the child's socio-ecological context, such as open family communication, child-centred parenting, and parents' knowledge regarding their children's activities. The authors also discuss how social support and neighbourhood and community cohesion may play a positive role in the development of a strong SOC in adolescence.

\section{Transitions in Adulthood}

Salutogenic research with adults has focused on their roles in workplaces, families, communities, and caregiving institutions. Researchers have tended to neglect the lifetime and developmental perspective of adult age. This might reflect a general view of the adult as a performing, not as a developing individual. It might be, as well, a consequence of methodological difficulties in describing transitions at various points of life in biographies that may deviate from a formerly "normal" life course, becoming more and more diverse.

Nevertheless, lifespan developmental psychology of the past two decades provides an understanding of adulthood as a series of interrelated transitions (Sugarman, 2001). Transition periods have been shown to play a crucial role in one's adaption to life stressors, and the development of physical and mental health throughout the life course.

In Chap. 17, Claudia Meier Magistretti and Beat Reichlin treat the topic of the transition from adolescence to adulthood and focus on young adults facing difficulties during this transition. Young adults not in education, employment, or training (NEETs) emerged only recently as an interest in public health and health promotion debate. Although estimates of the number of NEETS vary, the problem seems to be global and growing. This has stimulated the International Labour Organisation to talk in terms of "a generation at risk". Yet, the many programs that try to support NEETs fail to reach them and guide them successfully into work, housing, and social participation.

The authors consider why current initiatives to assist NEETs do not produce sustainable effects. "Helping" systems regard NEETs as patients, cases, or not yet enabled adults and thereby fail to meet their needs as well as their potential. Confronting the limits of current approaches, the authors emphasise the need for a salutogenic orientation in research and practice with NEETs. To provide guidance and inspiration in this direction, the authors describe new and promising NEET initiatives. These initiatives are characterised by having a genuine health orientation, NEET participation, the centrality of learning processes, and flexible, adaptive models of individual and social development in combination with enhanced employment, education, training, and entrepreneurship opportunities.

In Chap. 18, Ottomar Bahrs and colleagues focus on the transition from middle to late adulthood. The questions of when and how past transitions affect subsequent aging are discussed. While middle age was long considered undramatic, the authors state that it is increasingly gaining profile. They focus on a phase typically observed in the sixth decade of life, characterised by the initiation of the transition to deprofessionalisation and change of responsibilities within the family when crisis and chronic situations can lead to the need for help from health professionals.

Bahrs and colleagues deliberate on how a dialogue between middle-aged adults and professionals can contribute positively to the naming, modification, design, and further development of health goals, linking physiological and unconscious processes to the theory of salutogenesis.

Notably, the authors frame illness processes from the perspective of salutogenic resources, also discussing the benefits of crises experienced in middle adulthood.

I hope the authors' novel ideas about a salutogenic perspective on life in middle adulthood will inspire researchers and practitioners to a more innovative approach to this large segment of society.

\section{Older Age}

In Chap. 19, Maria Koelen and Monica Eriksson consider the meanings of the concepts of healthy aging, aging well, salutogenic aging, and reciprocity between the SOC and aging processes. They discuss how the community can pro- 
vide resources to strengthen older adults' SOC, perceived well-being, and quality of life. Quoting "It's not how old we are; it's how we are old", the authors illuminate critical differences in understanding healthy aging by professionals, researchers, and older people themselves.

While professionals tend to focus on negatively phrased topics (e.g. disability, disease, loneliness), older people emphasise a supportive social environment, the ability to use resources, to manage restrictions, and to make one's own decisions. As the authors clarify, evidence on the potential for enhanced health at older age points clearly to these factors: participation in social networks, contact with family and friends, and engagement in leisure and social activities. Since many of the prerequisites to strengthen general resistance resources (GRRs), specific resistance resources (SRRs), and the SOC are provided by or mediated through the community, the authors emphasise the role of intergenerational and age-friendly communities in providing supportive neighbourhood environments, social cohesion, and adequate transportation facilities for older people, and support for their ability to continue living in their own homes.

\section{Enhancing the Sense of Coherence along the Life Span}

Chapter 20, by Eva Langeland and colleagues, is titled Effectiveness of interventions to enhance the sense of coherence in the life course: a summary of the empirical evidence. It provides an overview of the effectiveness of programs and interventions that aim to strengthen the SOC throughout the lifespan. The authors report on more than 40 studies investigating the effectiveness of interventions to strengthen the SOC. They structure and summarise findings for young people, employed and unemployed adults, health professionals, people with disabilities, people with psychosomatic and mental health problems, people with physical problems, hospital patients, and older people. Even though most studies are limited to short-term pre-test and post-test study designs, a few studies have investigated the effects of intervention for longer follow-up periods of up to several months, and even more than 1 year.

The authors classified interventions on the extent of their salutogenic orientation, defining criteria for salutogenic interventions based on Antonovsky's theory and current research. They found that the effective interventions aiming at strengthening the SOC might have three qualities: they facilitate access and use of GRRs and SRRs, they foster active participation, and they understand SOC development as a learning process. The authors conclude that more intervention studies are needed, with (a) a more robust grounding in salutogenesis principles, (b) larger sample sizes, (c) longer follow-up periods, and (d) stronger research designs. Overall, the chapter indicates that it is possible to improve the SOC throughout the whole life course and that health promotion professionals' work to enhance the SOC should embrace a person-centred, resource-oriented approach.

\section{Knowledge, Gaps, and Perspectives}

In closing, we return to the three questions posed at the start of this chapter:

- What are the crucial factors that contribute to build, enhance, and strengthen the SOC during the life course?

- Is the role of the SOC in coping stable during the life course, or does it have different functions at different life stages? For example, does the SOC open one to new GRRs at the beginning and facilitate access to GRRs later in life?

- Can salutogenic interventions strengthen the SOC during various phases in the lifespan?

Addressing the first question on crucial factors that contribute to a strong SOC in the various phases of the life course, the authors of these chapters are unanimous about the significance of a strong linkage between the development of the SOC and the availability and use of GRRs. Even though GRRs change during a lifespan, the family, the social circle, and the community environment are crucial to the development of a strong SOC from early life to old age. At all life stages, the SOC is the product of the interaction of an individual and the environment - of the SOC, GRRs, and SRRs.

Therefore, the enrichment, attainability, and accessibility of resources are crucial to strengthening the SOC. This conclusion, based on mounting evidence presented in these chapters, is supportive of Antonovsky's contention that the most significant potential for strengthening the SOC lies in the availability and use of resistance resources.

Yet, as he regretfully noted, fostering resistance resources in a significant way depends on "radical change in the institutional, social, and cultural settings that shape people's life experiences" (Antonovsky, 1987, p. 124). Those levels of social life are rarely subjects of health research and not a primary focus of the chapters discussed here. However, this subject is taken up in other parts of this Handbook, most notably in Chap. 24 on the application of salutogenesis in politics and policy-making.

The answer to the second question is that we lack sufficient evidence to comment decisively about the stability of the SOC during the life course and its functions at dif- 
ferent ages. There is a striking lack of research on healthy adults and little research on weak SOC and how to strengthen it. Questions of how a strong SOC is created in children are partly answered, but we do not know how a strong SOC is created and maintained in adulthood, and we do not know how stable positive changes in people's SOC are over the lifetime. This gap might be due to the view of the adult as a performer of different roles, rather than as an individual in development. Therefore, salutogenic research investigates adults mainly as employees, parents, patients, caregivers, or inhabitants of cities or communities.

The third question, on how to strengthen the SOC, can be answered to a degree. It seems the SOC can be influenced at any age and in many life situations, as Chap. 20 on salutogenic interventions discusses. Antonovsky suggested that lasting SOC strength is obtained when interventions enable people to perceive coherent life experiences, when they learn how to nourish their sense of comprehensibility, meaningfulness, and manageability.

\section{References}

Antonovsky, A. (1985). The life cycle, mental health and the sense of coherence. Israel Journal of Psychiatry \& Related Sciences, 22(4), 273-280.

Antonovsky, A. (1987). Unraveling the mystery of health: How people manage stress and stay well. Jossey-Bass.

Erikson, E. (1950). Growth and crises of the healthy personality. Psychological Issues, 1, 50-100.

Lindström, B., Berg, M., Meier Magistretti, C., Perez-Botella, M., \& Downe, S. (2017). The salutogenic approach to maternity care from theory to practice and research. In Sinclair et al. (Eds.), New thinking on improving maternity care. Pinter \& Martin Ltd.

Lindström, B., \& Eriksson, M. (2010). The Hitchhiker's guide to salutogenesis: Salutogenic pathways to health promotion. Helsinki.

Maass, R., Lindstrom, B., \& Lillefjell, M. (2017). Neighborhoodresources for the development of a strong the SOC and the importance of understanding why and how resources work: A grounded theory approach. BMC Public Health, 17(1), 704.

Montessori, M. (1995). The absorbent mind. Henry Holt \& Co.

Sugarman, L. (2001). Lifespan development. Frameworks, accounts, and strategies (2nd ed.). Psychology Press.

Open Access This chapter is licensed under the terms of the Creative Commons Attribution 4.0 International License (http://creativecommons. org/licenses/by/4.0/), which permits use, sharing, adaptation, distribution and reproduction in any medium or format, as long as you give appropriate credit to the original author(s) and the source, provide a link to the Creative Commons license and indicate if changes were made.

The images or other third party material in this chapter are included in the chapter's Creative Commons license, unless indicated otherwise in a credit line to the material. If material is not included in the chapter's Creative Commons license and your intended use is not permitted by statutory regulation or exceeds the permitted use, you will need to obtain permission directly from the copyright holder. 